\title{
Antioxidant Properties and Stability of Geissospermum Reticulatum Tinctures: Lag Phase ESR and Chemometric Analysis
}

\author{
J.J. Sajkowska-Kozielewicz*, K. Gulik, K. Makarova and K. Paradowska \\ Department of Physical Chemistry, Faculty of Pharmacy, Medical University of Warsaw, \\ S. Banacha 1, 02-097 Warsaw, Poland
}

\begin{abstract}
Geissospermum species are widely used in folk medicine in the Amazon region. This study was conducted to determine total phenolic and flavonoid contents of three tinctures of Geissospermum reticulatum barks from Peruvian Amazon and correlate these contents to the antioxidant activities and stability. Total content of phenolic compounds (from 694.91 to $1430.67 \mathrm{mg} \mathrm{GAE} / \mathrm{kg}$ ) and flavonoids $(575.23-815.65 \mathrm{mg} \mathrm{CAE} / \mathrm{kg}$ ) were found by spectrophotometric methods. The obtained values were interpreted by artificial neural networks to describe the most beneficial conditions for tinctures. All tinctures have demonstrated the maximum of total flavonoid between 14 and 20 weeks of maceration, whereas the maximum of total flavonoid was between 25 and 30 . The highest antioxidant properties were exhibited by tinctures in 3 different tests (ferric reducing ability of plasma, DPPH-ESR, oxygen radical absorbance capacity) after 35 weeks of maceration. The principal component analysis was employed to relate contents and properties. Results from the lag phase with $\alpha$-(4-pyridyl-1-oxide)-N-tert-butylnitrone (POBN) spin trap studies at $60^{\circ} \mathrm{C}$ demonstrated that the stability of tinctures were related to total phenolic content. Thus, samples with 550-800 mg GAE/ $\mathrm{kg}$ were more stable than those with higher total phenolic contents. The most beneficial conditions for bark tinctures depend on aimed final products, e.g. maximum of polyphenols or flavonoids and long-term stability. Further studies about content and storage conditions are needed.
\end{abstract}

DOI: 10.12693/APhysPolA.132.68

PACS/topics: 07.05.Mh, 87.64.kh, 87.80.Lg

\section{Introduction}

In the recent years there has been a growing interest in the use of plants from the South America. This is because plants from the Amazon region exhibit wide range of medical properties $[1-5]$ and can be investigated more efficiently [6]. For centuries people believed in the healing properties of traditional remedies, e.g. tinctures and infusions, and were preparing them at home. Today we have advanced methods to study properties of these products.

Native people of Amazon region widely use barks and leaves of Geissospermum species for numerous medicinal purposes. There are some evidence showing that Geissospermum species demonstrate anti-malarial, antibacterial and nociceptive activities $[1,7,8]$. In our previous publication we reported that infusions, tinctures and extracts of Geissospermum reticulatum bark have potential to be used as good sources of bioactive compounds and exhibit antioxidant, cytotoxic and anti-proliferative properties [9]. However, there are still some questions about content and storage conditions of tinctures, which are one of the most popular serving forms because of their taste and medical activities.

In the present study we used the fluometric oxygen radical absorbance capacity (ORAC) assay, the 2,2-diphenyl1-picrylhydrazyl (DPPH) radical scavenging method and

*corresponding author; e-mail: jsajkowska@gmail.com the ferric reducing ability of plasma (FRAP) assay to test antioxidant properties of tinctures of three samples of Geissospermum reticulatum barks during maceration. We also defined the total polyphenol (TP) and flavonoid (TF) contents of tinctures by spectrophotometric methods and interpreted the obtained values using artificial neural networks (ANN) to find the most beneficial conditions for these products. To relate contents and properties we employed the principal component analysis (PCA) which has been used in other studies [10]. The stability of studied tinctures was assessed using lag phase electron spin resonance (ESR) with $\alpha$-(4-pyridyl1-oxide)-N-tert-butylnitrone (POBN) spin probe, which had been previously used in the studies of beer $[11,12]$ and wine [13]. Application of all these methods allowed us to determine that short-term preparation and storage under low-light conditions are beneficial to the tinctures. Our approach can be used to prepare other plant-based products.

\section{Materials and methods 2.1. Plant materials}

Three samples of dried barks of Geissospermum reticulatum were a kind gift from National Agrarian University - La Molina (Lima, Peru). Barks were collected in Peruvian Amazon in June 2013 in obedience to local regulations.

\subsection{Preparation of tinctures of G. reticulatum}

Tinctures were prepared by adding $25 \mathrm{~mL}$ of $70 \%$ ethanol to $5 \mathrm{~g}$ of cut dry bark. The samples were collected in the 1st, 4th, 6th, 35th, and 48th week of macerations. 


\subsection{Bioactive compounds}

The modified Folin-Ciocalteu colorimetric method [14] was applied to determine total polyphenol (TP) contents. Gallic acid was used to calculate the calibration curve $\left(50-500 \mathrm{mg} / \mathrm{L} ; y=0.001 x+0.015, R^{2}=0.99\right)$ and $\mathrm{TP}$ was expressed as gallic acid equivalents ( $\mathrm{mg}$ GAE $/ \mathrm{kg}$ ). Total flavonoid (TF) contents were determined according to Park et al. protocol [15]. TF were expressed as means $\mathrm{mg}$ of quercetin equivalent $(\mathrm{mg} \mathrm{CAE} / \mathrm{kg}, 0.05-$ $\left.0.4 \mathrm{mg} / \mathrm{mL}, y=1.408 x-0.003, R^{2}=0.99\right)$. Details are submitted in our previous publication [9]. All experiments were performed in triplicates.

\subsection{Antioxidant activity}

The ORAC was measured following the $\mathrm{Ou}$ et al. method [16], slightly modified by Číž et al. [17]. The FRAP assay was based on that proposed by Benzie and Strain [18]. The free radical scavenging activity of the tinctures was measured in vitro by 2,2 '-diphenyl1-picrylhydrazyl (DPPH) assay following the BrandWilliams et al. method [19]. All experiments were performed in triplicates.

\subsection{Lag phase ESR}

The Fenton reaction was used to generate free radicals. POBN ( $\alpha$-(4-pyridyl-1-oxide)-N-tert-butylnitrone) spin trap was purchased from Sigma-Aldrich Chemicals (Poznań, Poland). Ferrous sulfate $\left(\mathrm{FeSO}_{4} \cdot 7 \mathrm{H}_{2} \mathrm{O}\right)$ and ethanol were obtained from POCh (Gliwice, Poland). For the Fenton reaction in tinctures we added respectively: $20 \mathrm{mM}$ POBN $(20 \mu \mathrm{L}), 1 \mathrm{mM} \mathrm{FeSO}_{4}(20 \mu \mathrm{L})$ and the studied sample $(40 \mu \mathrm{L}$ of a tincture or a reference solution). Then the sample was vortexed (10 s) and the first measurement was performed $90 \mathrm{~s}$ after the addition of sample. The next 59 measurements were performed every $90 \mathrm{~s}$. The following concentrations of gallic acid were used as references: 200, 400, and $1400 \mathrm{mg}$ $\mathrm{GAE} / \mathrm{kg}$. As references for the behavior of flavonoids the following concentrations of catechin was used: 400 and $800 \mathrm{mg} \mathrm{CAE} / \mathrm{kg}$. All these solutions were prepared in $70 \%$ ethanol. The optimization of the lag phase experimental conditions was performed at $55^{\circ} \mathrm{C}, 60^{\circ} \mathrm{C}$ and $70^{\circ} \mathrm{C}$. Further experiments were performed in duplicates at $60^{\circ} \mathrm{C}$.

\subsection{EPR measurements}

EPR measurements were performed on $\mathrm{X}$ band (9.5 GHz) MiniScope200 spectrometer (Magnettech, Germany). For DPPH measurements we used central field $334.3 \mathrm{mT}$, sweep $7.1 \mathrm{mT}$, sweep time $20 \mathrm{~s}$, modulation amplitude $0.1 \mathrm{mT}$, microwave power $8 \mathrm{~dB}$. For lag phase we used sweep time $20 \mathrm{~s}$, modulation amplitude $0.05 \mathrm{mT}$, microwave power $10 \mathrm{~dB}$, sweep $6.9 \mathrm{mT}$. DPPH signal and POBN spin adduct signal were not saturated. Simulations of ESR spectra were performed in Matlab, using EasySpin toolbox with "garlic" function in order to minimize the error in the second integral. Each spectrum was simulated as a superposition of two spectra from POBN radical adducts, e.g. $5 \%$ of hydroxyl and $95 \% \mathrm{C}$-centered radical adduct correspondently.

\section{7. $P C A$}

PCA has been performed to examine the qualitative difference between three samples of bark (B1, B2, and B3). Samples were measured three times in each experiment $(1,4,6,35$, and 48 weeks). The non-averaged data of total polyphenol and flavonoid contents and antioxidant capacity (ORAC, DPPH, FRAP) was analyzed in MATLAB (The Mathworks Inc., Natick, MA, USA) using "princom" function.

\section{8. $A N N$}

ANN was used to predict changes of flavonoid and polyphenol contents with time. Multilayer perceptron with 1 hidden layer was used. The log-sigmoid transfer function (logsig) was used. The input data were type of sample (1 to 3 ) and week of measurement $(1,4,6$, 35 , and 48 weeks). All input data were normalized to $0: 1$ range. The output data were contents of polyphenols and flavonoids. The programs and algorithms used for the ANN modelling were developed in MATLAB (The Mathworks Inc., Natick, MA, USA).

\subsection{Statistical analysis}

The results of phenolic and flavonoid contents and antioxidant activity (TP, TF, ORAC, DPPH, FRAP) are presented as mean. Statistical analyses were performed using GraphPad Prism 6 for Windows (GraphPad Software Inc., version 6.01, San Diego, USA). Differences between means were determined by analyses of variance (one way ANOVA) followed by Tukey's honest significant difference (HSD) test with $\alpha=0.05$.

\section{Results and discussion}

\subsection{Total flavonoid and polyphenol contents}

Plant phenolics constitute one of the major groups of compounds acting as primary antioxidants. The amount of TP in the studied tinctures varied from 694.91 to $1430.67 \mathrm{mg} \mathrm{GAE} / \mathrm{kg}$ (Table I). The highest TP content was found in tinctures after 35 weeks of maceration for all samples. The next highest values were observed after 6 weeks. The TF, a subgroup of polyphenols, contents were also measured (Table I). Again the highest TF contents were observed for tinctures after 35 weeks of maceration (from 575.23 to $815.65 \mathrm{mg} \mathrm{CAE} / \mathrm{kg}$ ) followed by 6 weeks values (from 545.81 to $726.09 \mathrm{mg} \mathrm{CAE} / \mathrm{kg}$ ). The values for TP and TF contents in tinctures after 1 week and also 48 weeks of maceration were significantly lower. Thus, the time of maceration is an important aspect of extraction of bioactive compounds and then their stability.

\subsection{Antioxidant properties}

The antioxidant effect of plant-based samples can be determined by a few different in vitro methods as antioxidants present in natural products can deactivate radicals by two major mechanisms: hydrogen atom transfer (HAT) and single electron transfer (SET) [20]. That is why to establish the antioxidant properties of plantbased products, a combined assay of several tests is required [21]. The FRAP assay is the example of the SET 


\section{TABLE I}

Antioxidant properties of $G$. reticulatum tinctures. Values are the mean of three replicates. The data followed by the same superscript letter are not significantly different. Time in weeks was counted from the start of maceration. A - polyphenols [mg GAE $/ \mathrm{kg}$ ], B — flavonoids [mg CAE $/ \mathrm{kg}$ ], C ORAC [mmol TE/L], D - FRAP $[\mathrm{mmol} / \mathrm{L}], \mathrm{E}-$ $\mathrm{DPPH}[\mathrm{mg} \mathrm{DPPH} / \mathrm{L}]$.

\begin{tabular}{c|c|c|c|c|c|c}
\hline \hline Bark & Time & $\mathrm{A}$ & $\mathrm{B}$ & $\mathrm{C}$ & $\mathrm{D}$ & $\mathrm{E}$ \\
\hline \multirow{4}{*}{1} & 1 & 553.64 & 471.25 & 22.75 & 3.75 & 1.48 \\
\cline { 2 - 7 } & 4 & 590.45 & 513.01 & 24.44 & 3.82 & 1.78 \\
\cline { 2 - 7 } & 6 & 828.59 & 545.81 & $32.34^{b}$ & 4.92 & 2.14 \\
\cline { 2 - 7 } & 35 & 896.22 & 604.35 & 51.33 & 6.02 & 3.59 \\
\cline { 2 - 7 } & 48 & 364.91 & $371.46^{a}$ & 35.11 & 5.36 & 2.31 \\
\hline \multirow{5}{*}{2} & 1 & 754.36 & 572.68 & 36.53 & 5.27 & 0.90 \\
\cline { 2 - 7 } & 4 & 832.64 & 798.32 & 43.89 & 5.67 & 2.66 \\
\cline { 2 - 7 } & 6 & 1227.85 & 726.09 & 63.48 & 6.73 & 2.89 \\
\cline { 2 - 7 } & 35 & 1430.67 & 815.65 & 73.63 & 7.46 & 4.37 \\
\cline { 2 - 7 } & 48 & 983.18 & 526.87 & 63.27 & 6.64 & 3.46 \\
\hline \multirow{5}{*}{3} & 1 & 448.09 & 386.02 & 20.91 & 2.59 & 0.45 \\
\cline { 2 - 7 } & 4 & 608.73 & 570.54 & 33.65 & 3.47 & 1.56 \\
\cline { 2 - 7 } & 6 & 678.44 & 568.13 & $45.17^{b}$ & 4.73 & 2.46 \\
\cline { 2 - 7 } & 35 & 694.91 & 575.23 & 59.47 & 4.82 & 3.82 \\
\cline { 2 - 7 } & 48 & 615.26 & $361.19^{a}$ & 27.23 & 3.72 & 1.74
\end{tabular}

reaction, whereas ORAC and DPPH assays are based on the HAT reaction. In many studies on antioxidants the spectrophotometric (UV-vis) method with the DPPH radical have been employed. However, the use of ESR is preferred when samples are cloudy and colored as we have it in this work.

The highest antioxidant properties were defined in 3 different tests for the tinctures after 35 weeks of maceration which corresponds to maximum of TP and TF contents (Table I). Again, 6 weeks values were slightly lower. That could indicate that maximum of content and properties occurred between 6 and 35 weeks of maceration.

\section{3. $P C A$}

PCA was performed in order to visualize the results from Table I and to explore the dependence between the content and the antioxidant activity in the studied samples. The first three PCA components describe $95 \%$ of the sample variation. The biplot clearly shows that TF and TP content are weakly correlated with the antioxidant properties (angles between eigenvectors are close to $90^{\circ}$ ) (Fig. 1), whereas the results of all antioxidant methods are highly correlated, even though DPPH, ORAC and FRAP are aimed on different mechanism of actions. The PC1 gives poor separation of samples, but all samples are well distributed along PC2. Moreover, some similarity between 1st (B1) and 3rd (B3) sample of bark could be spotted (Fig. 1), whereas the 2nd sample (B2) clearly stands out.

When PC2 and PC3 are considered, all samples could be grouped according the time of maceration (Fig. 2). PCA showed that after 1 and 6 weeks of macerations

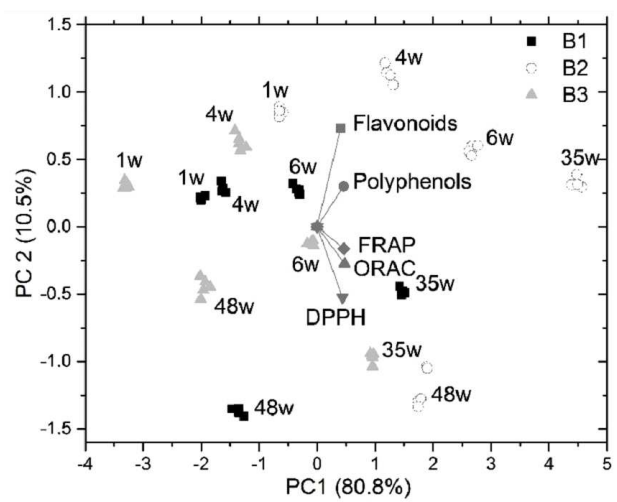

Fig. 1. Biplot of the first two principal components (PC1 and $\mathrm{PC} 2$ ) of B1, B2, B3 tincture samples at 1, $4,6,35$, and 48 weeks.

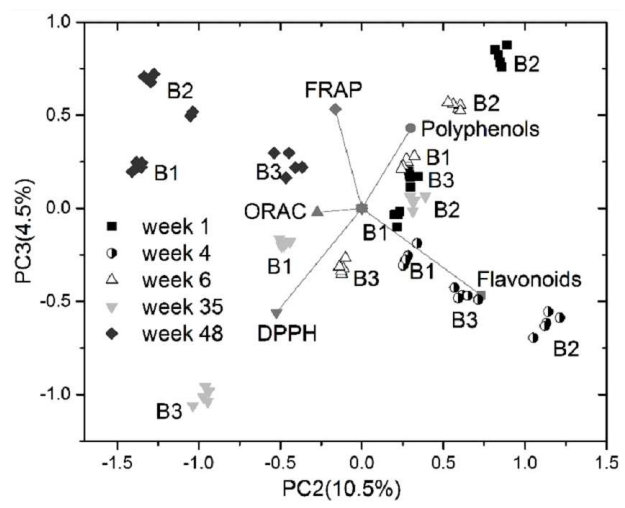

Fig. 2. Biplot of the first two principal components (PC2 and $\mathrm{PC} 3)$ of $\mathrm{B} 1, \mathrm{~B} 2, \mathrm{~B} 3$ tincture samples at 1, $4,6,35$, and 48 weeks.

the most changes occurred in polyphenol content. After 4 weeks of maceration the flavonoid content had the greatest impact of the sample variation. The DPPH values variation was dominant at 35 weeks of maceration, whereas after 48 weeks of maceration the samples varied by ORAC and FRAP values. In order to explain these variations and influence of the changes of TF and TP on antioxidant properties of the tincture more experiments at different time points are required.

\section{4. $A N N$}

ANN are used as an accurate function approximation [22]. When ANN is well trained, it could approximate the values between the experimental points. Here the ANN was created to approximate the TF and TP values at additional points of time e.g. from 1 to 48 weeks of maceration with time step 1 week. Our multilayer perceptron required about 22000 epochs to train. The validation of the created ANN on the testing set showed the very high correlation $(R=0.98)$ with our experimental results. The results of ANN approximation (Figs. 3, 4) revealed that the maximum of TP did not correspond to the maximum of TF. The maximum value of polyphenols content appeared after 15-20 weeks of maceration, while the maximum of flavonoids content was predicted 
to be after 25-35 weeks of maceration. The decrease of TP content after 20 weeks could be explained by degradation of the compounds which were extracted by $70 \%$ ethanol. Whereas flavonoid extraction could be slower, and thus maximum is observed approximately $5-10$ weeks later. These processes could play important role in the stability of the tincture.

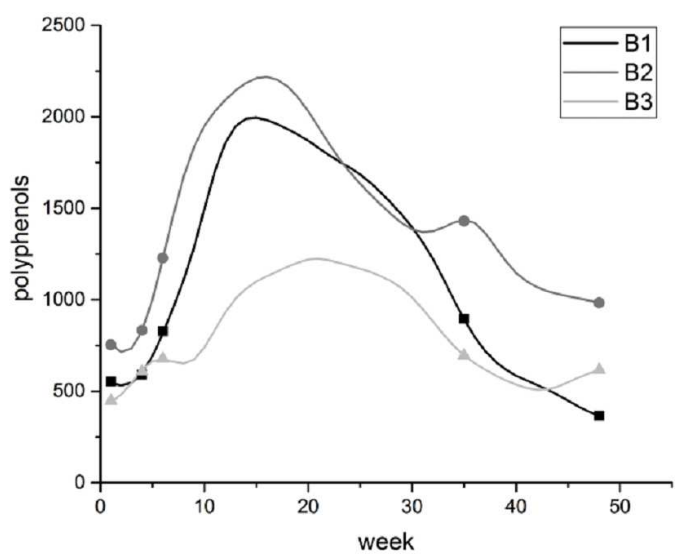

Fig. 3. Results of ANN predictions of polyphenol content as a function of maceration time for tinctures prepared from bark 1,2 , and 3 . The maximum values of polyphenol contents appeared in 15th-20th weeks for all figures.

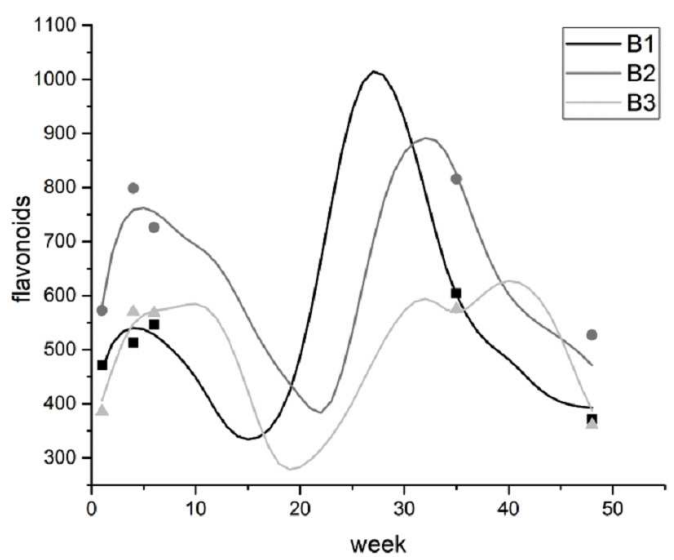

Fig. 4. Results of ANN predictions of flavonoid content as a function of maceration time for tinctures prepared from bark 1,2, and 3 . The maximum values of flavonoid contents was in 25 th-35th weeks for all tinctures.

\subsection{Lag phase ESR}

Lag phase ESR is widely used in beer and wine stability studies [11-13]. In this work we explored its application to stability studies in tinctures.Data represented as a mean from 2 independent experimentsData represented as a mean from 2 independent experimentsData represented as a mean from 2 independent experimentsData represented as a mean from 2 independent experiments

As the first step we optimized the temperature of the lag phase experiments. No radical adducts were formed

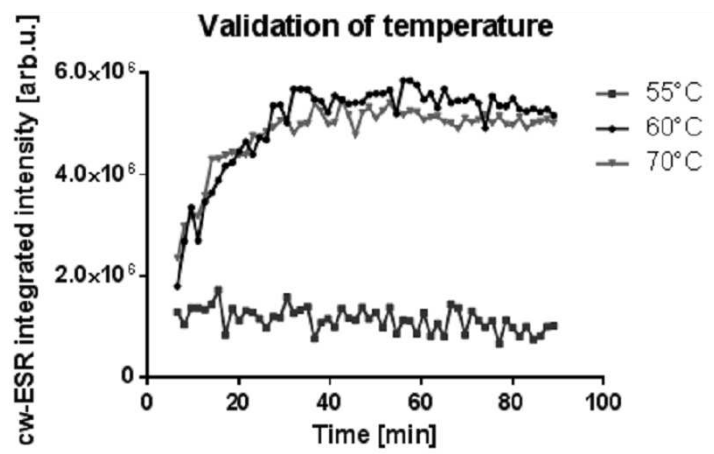

Fig. 5. The integrated intensity of ESR signals of POBN spin adducts at different temperature.

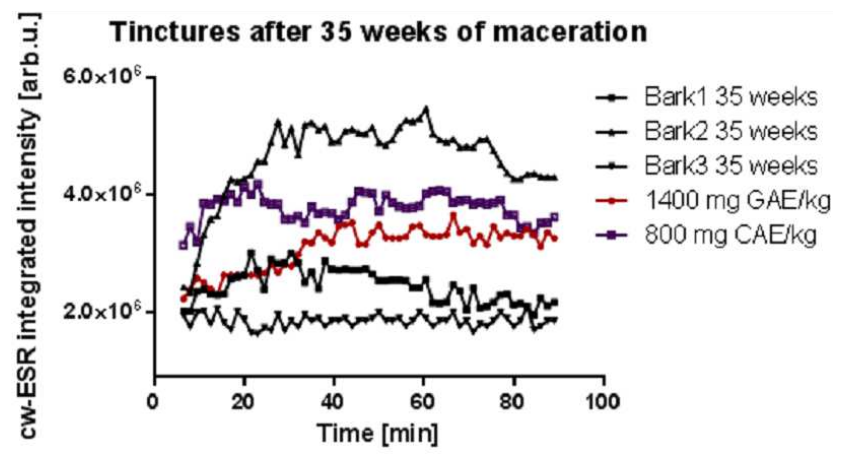

Fig. 6. The integrated intensity of ESR signals of POBN spin adducts after 35 th week of maceration.

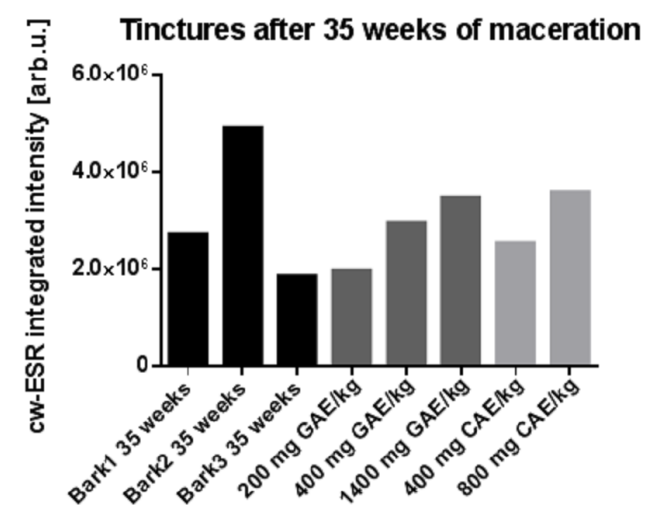

Fig. 7. The integrated intensity of ESR signals of tinctures at $41 \mathrm{~min}$ of the experiment at $60^{\circ} \mathrm{C}$.

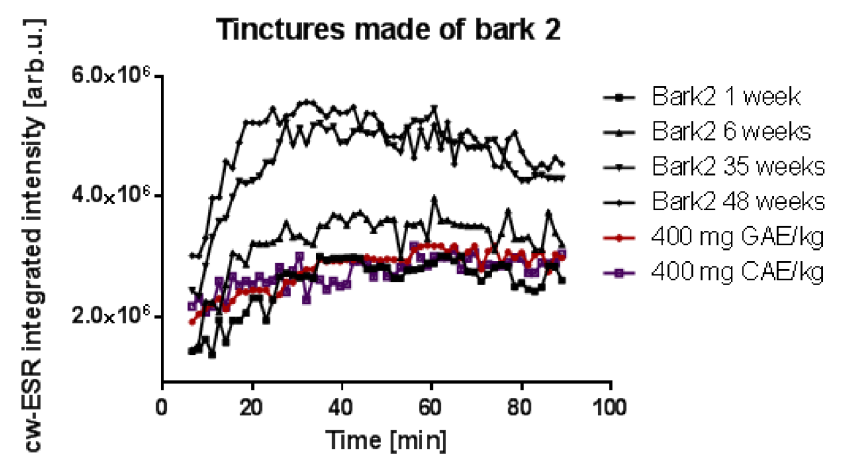

Fig. 8. Spectra of POBN spin adducts of tinctures of bark 2 . 
at $55^{\circ} \mathrm{C}$. At $60^{\circ} \mathrm{C}$ and $70^{\circ} \mathrm{C}$ the ESR signal from POBN adducts occurred (Fig. 5). Both samples showed similar kinetics, thus only $60^{\circ} \mathrm{C}$ was considered for further studies.

As the maximum of TP, TF and antioxidant properties were observed after 35 weeks of maceration, we compared the radical adduct production of $3 \mathrm{G}$. reticulatum tinctures with the reference solutions of $1400 \mathrm{mg} \mathrm{GAE} / \mathrm{kg}$ and $800 \mathrm{mg} \mathrm{CAE} / \mathrm{kg}$ (Fig. 6). The most representative ESR signal intensities were at $41 \mathrm{~min}$ of the experiment. When comparing the ESR signals at that time, tinctures made of bark 1 and 3 had lower intensities than those of corresponding reference solutions (Fig. 7). On the other hand, the tincture made of bark 2 had significantly higher ESR signal intensity which indicated high radicals formation. That indicates that pure compounds could act in a different way than mixture of them. Thus, in case of 1 and 3 tinctures, the overall ESR signal was lower due to their higher stability. That could be explained by the presence of other type of bioactive compounds with high antioxidant effect. In case of tincture from bark 2, the too high initial level of TP and TF could decrease the stability of the sample and lead to high rate of radicals adduct formation. That is probably the case of the tincture from bark 2 after 48 weeks of maceration. The kinetics of the ESR signal in lag-phase experiment is similar to those of the sample after 35 weeks of maceration (Fig. 8). However, tinctures with shorter time of maceration, e.g. 1-6 weeks, appeared to be more stable as their kinetics are close to those of the $400 \mathrm{mg} \mathrm{CAE} / \mathrm{kg}$ and $400 \mathrm{mg} \mathrm{GAE} / \mathrm{kg}$ references.

Our data suggest that TP and TF contents do not explain completely the antioxidant activities in plantbased mixtures. Moreover, tinctures rich in polyphenols and flavonoids could be less stable during the long-term storage.

\section{Conclusions}

We can demonstrate that by using this data analysis approach it is possible to select experiments which carry more information. Thus, we have been able to determine that short-term preparations (6-12 weeks) and storage under low-heat conditions (at less than $55^{\circ} \mathrm{C}$ ) are beneficial to the tinctures. Our methods can be therefore used to optimize other plant-based products.

\section{Acknowledgments}

This project was financially supported by Medical University of Warsaw research fellowships for Young Scientists (No. FW28/PM2/16). The barks of G. reticulatum were a kind gift from National Agrarian University — La Molina (Lima, Peru).

\section{References}

[1] B. Gordillo-Román, M. Reina, L. Ruiz-Mesia, W. Ruiz-Mesia, P. Joseph-Nathan, Tetrahedron Lett. 54, 1693 (2013).
[2] A.K. Machado, A.C. Andreazza, T.M. Da Silva, A.A. Boligon, V. Do Nascimento, G. Scola, A. Duong, F.C. Cadoná, E.E. Ribeiro, I.B.M. Da Cruz, Oxid. Med. Cell. Longev. 2016, 8940850 (2016).

[3] A.I.T.D. Oliveira, T.S. Mahmoud, G.N.L.D. Nascimento, J.F.M.D. Silva, R.S. Pimenta, P.B.D. Morais, Sci. World J. 2016, 9734181 (2016).

[4] P. Kozielewicz, K. Paradowska, S. Erić, I. Wawer, M. Zloh, Monatsh. Chem. 145, 1201 (2014).

[5] P.P.D.T. Espindola, P.D.S.D. Rocha, C.A. Carollo, W.O. Schmitz, Z.V. Pereira, M.D.C. Vieira, E.L.D. Santos, K. de Picoli Souza, Oxid. Med. Cell. Longev. 2016, 7910340 (2016).

[6] Y.W. Mak, L.O. Chuah, R. Ahmad, R. Bhat, J. King Saud Univ. Sci. 25, 275 (2013).

[7] M.J.S. Vital, A.L.B. Carneiro, L.F. Rocha, R.C.D.N. Amorim, M.R.M. Camargo, A.M. Pohlit, Rev. Fitos Eletronica 8, 137 (2013).

[8] M. Reina, W. Ruiz-Mesia, M. López-Rodriguez, L. Ruiz-Mesia, A. González-Coloma, R. MartinezDiaz, J. Nat. Prod. 75, 928 (2012).

[9] J.J. Sajkowska-Kozielewicz, P. Kozielewicz, N.M. Barnes, I. Wawer, K. Paradowska, Oxid. Med. Cell Longev. 2016, 2573580 (2016).

[10] M.K. Jamróz, K. Paradowska, K. Zawada, K. Makarova, S. Kaźmierski, I. Wawer, J. Sci. Food Agr. 94, 246 (2014)

[11] N.M. Kocherginsky, Y.Y. Kostetski, A.I. Smirnov, J. Agric. Food Chem. 53, 6870 (2005).

[12] A.M. Frederiksen, R.M. Festersen, M.L. Andersen, J. Agric. Food Chem. 56, 8514 (2008).

[13] R.J. Elias, M.L. Andersen, L.H. Skibsted, A.L. Waterhouse, J. Agric. Food Chem. 57, 4359 (2009).

[14] V. Singleton, R. Orthofer, R.M. Lamuela-Raventós, Method. Enzymol. 299, 152 (1999).

[15] Y.-S. Park, S.-T. Jung, S.-G. Kang, B.G. Heo, P. Arancibia-Avila, F. Toledo, J. Drzewiecki, J. Namiesnik, S. Gorinstein, Food Chem. 107, 640 (2008).

[16] B. Ou, M. Hampsch-Woodill, R.L. Prior, J. Agric. Food Chem. 49, 4619 (2001).

[17] M. Číž, H. Čížová, P. Denev, M. Kratchanova, A. Slavov, A. Lojek, Food Control 21, 518 (2010).

[18] I.F. Benzie, J.J. Strain, Anal. Biochem. 239, 70 (1996).

[19] W. Brand-Williams, M.E. Cuvelier, C. Berset, LWTFood Sci. Technol. 28, 25 (1995).

[20] R.L. Prior, X. Wu, K. Schaich, J. Agric. Food Chem. 53, 4290 (2005).

[21] J.H. Lee, S.J. Lee, S. Park, H.K. Kim, W.Y. Jeong, J.Y. Choi, N.-J. Sung, W.S. Lee, C.-S. Lim, G.S. Kim, S.C. Shin, Food Chem. 124, 1627 (2011).

[22] K. Hornik, M. Stinchcombe, H. White, Neur. Networks 2, 359 (1989). 